\title{
Three dimensional colour Doppler echocardiography for the characterisation and quantification of cardiac flow events
}

\author{
T Irvine, X N Li, R Rusk, D Lennon, D J Sahn, A Kenny
}

Cardiothoracic Unit, Freeman Hospital, Newcastle upon Tyne, UK

T Irvine

R Rusk

A Kenny

Oregon Health

Sciences University, Portland, Oregon,

USA

$\mathrm{XNLi}$

D J Sahn Laboratories, Bothell, Washington, USA

D Lennon

Correspondence to: Dr A Kenny, Regional Cardiothoracic Centre, Freeman Hospital, Newcastle upon Tyne NE7 7DN, UK antoinette.kenny@ncl.ac.uk
Advanced Technology

The accurate non-invasive quantification of cardiac flow volumes is an important goal in clinical cardiology. Computation of laminar volume flow through the ventricular outflow tracts would allow direct measurement of stroke volume and cardiac output. Grading of valvar regurgitation and follow up of patients with regurgitant lesions may be more accurately performed through calculation of regurgitant volume and fraction, and regurgitant orifice area. While estimates of these parameters can be made by conventional two dimensional (2D) echocardiographic techniques, these methods may be unreliable when applied to complex, dynamic, three dimensional (3D) flow events (table 1). ${ }^{1-13}$

The advent of three dimensional echocardiography (3DE) provides a solution to this basic limitation. 3DE generates a scan volume (as opposed to a two dimensional scan sector)

Table 1 Conventional 2D echo-Doppler cardiac flow quantification methods

\begin{tabular}{|c|c|}
\hline Technique & Limitations \\
\hline "Eyeball" grading of regurgitant jet & $\begin{array}{l}\text { Subjective } \\
\text { As for regurgitant jet dimensions }\end{array}$ \\
\hline $\begin{array}{l}\text { Regurgitant jet dimensions }{ }^{1-3} \text { (length, width, } \\
\text { area) }\end{array}$ & $\begin{array}{l}\text { Machine settings } \\
\text { Underestimation in the case of eccentric jets }\end{array}$ \\
\hline $\begin{array}{l}\text { Flow convergence region surface area } a^{4-6} \\
\text { (regurgitant volume, ROA) }\end{array}$ & $\begin{array}{l}\text { Morphological assumptions } \\
\text { (hemisperical/hemielliptical) }\end{array}$ \\
\hline Vena contracta cross sectional area ${ }^{7-9}(\mathrm{ROA})$ & $\begin{array}{l}\text { Angle dependency } \\
\text { Localisation }\end{array}$ \\
\hline $\begin{array}{l}\text { Quantitative combined spectral Doppler and } \\
\text { M/B mode methods }{ }^{10-13} \text { (regurgitant volume, } \\
\text { stroke volume) }\end{array}$ & $\begin{array}{l}\text { Cumbersome } \\
\text { Margin for error }\end{array}$ \\
\hline
\end{tabular}

ROA, regurgitant orifice area.

A

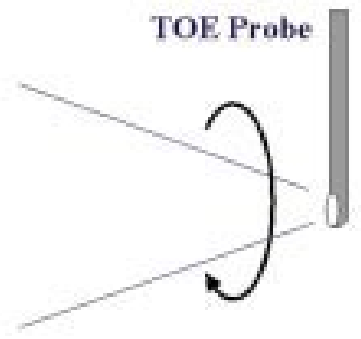

$180^{\circ}$ rotational acquisition $2-3^{\circ}$ step increments ECG \& respiratory gated

B

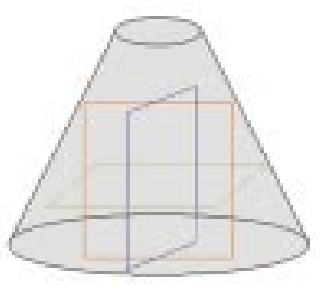

C

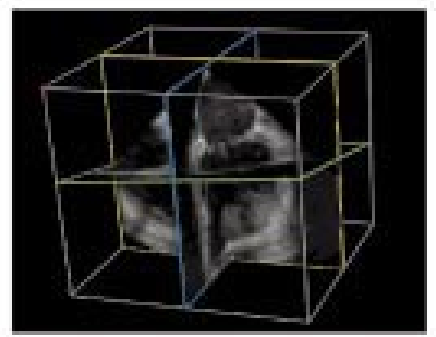

Figure 1 (A) Acquisition of multiple 2D images by rotation of multiplane transoesophageal probe through $180^{\circ}$. (B) Assimilation of multiple $2 \mathrm{D}$ imaging planes to generate a $3 D$ volume of data. Coloured lines show typical cutplanes within the dataset. $(C)$ Example of $3 D$ dataset with demonstration of cutplanes. which produces a three dimensional dataset containing entire cardiac structures. ${ }^{14} 15$

Surface rendering may then be performed to produce an image with feature contours and depth perspective. Such an approach has proved useful in the representation of complex cardiac structural pathology and in the generation of images which reflect typical "surgeon's eye" views of the heart. ${ }^{16}$ In addition, the $3 \mathrm{D}$ dataset may be transected by cut planes orientated in any direction within the volume ("anyplane imaging"). As the entire structure under study is encompassed in the dataset, volume calculations may be made without the need to make geometric assumptions regarding its morphology. A number of studies have confirmed the accuracy of $3 \mathrm{D}$ echocardiography for the calculation of cardiac chamber volumes. ${ }^{17-20} 3 \mathrm{D}$ flow quantitation methods are currently under development and it seems reasonable to assume that these will be superior to the 2D methods listed in table 1 . We review these new approaches to the quantification of cardiac flow events and indicate how they may ultimately be integrated into clinical practice.

\section{Technique of 3DE for the visualisation} and quantification of flow events

3D datasets may be generated essentially in two ways:

- By gated acquisition of multiple 2D echocardiographic planes, which are subsequently registered and integrated into a $3 \mathrm{D}$ block of data (dataset) by a dedicated 3DE reconstruction computer workstation ${ }^{2122}$

- Using a dedicated 3D echocardiographic imaging system, which interrogates a pyramidal scan volume and generates a $3 \mathrm{D}$ dataset in real time (real time three dimensional echocardiography (RT3D) $)^{23} 24$

Naturally, 3DE representation of flow events necessitates the incorporation of colour Doppler flow velocity information into the dataset. While RT3D colour Doppler echocardiography has recently been introduced, at present the suboptimal acoustic sampling rate available for colour Doppler severely restricts frame rates, colour Doppler quality, and hence clinical applicability; therefore we will not consider this method. Nevertheless, the principles outlined for 3D flow quantitation should be applicable to RT3D colour Doppler datasets in the future.

The most common gated acquisition method currently in use involves the rotation of a conventional 2D probe (either a multiplane probe or a conventional transthoracic probe mounted in an external rotation device) 

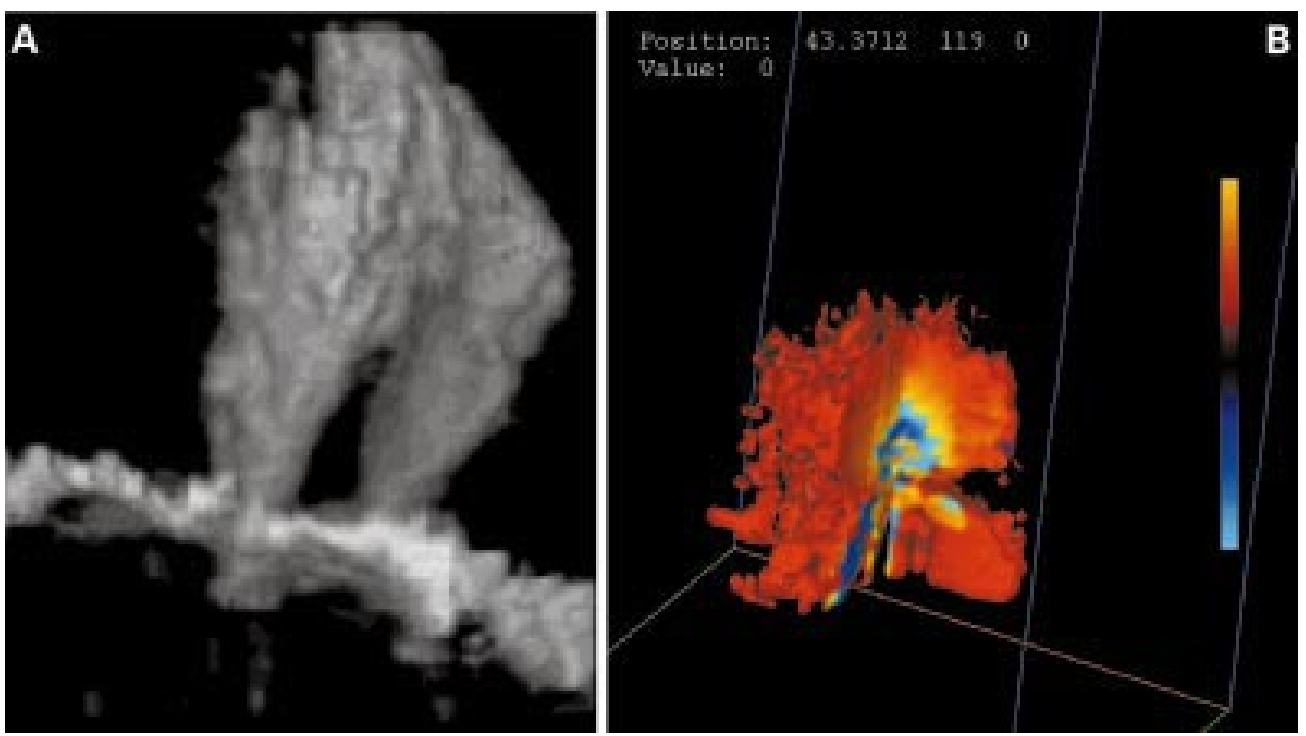

Figure 2 (A) Grey scale reconstruction of twin regurgitant jets extending upwards into the cavity of the left atrium. (B) Digital $3 D$ colour Doppler rendering of a flow convergence region (in vitro acquisition).

through $180^{\circ}$ around a central axis which is orientated perpendicular to the scan face. Multiple 2D images are then stored across the cardiac cycle (with ECG and respiratory gating) at fixed step increments over the $180^{\circ}$ sweep. Typically, increments of $2^{\circ}$ or $3^{\circ}$ are employed (fig 1). The $2 \mathrm{D}$ data may take the form of video composite data, transferred directly from the "video out" port on the echocardiographic system to the $3 \mathrm{D}$ reconstruction workstation, or they may be stored as digital data on board the ultrasound system itself. In either case, these $2 \mathrm{D}$ data are subsequently assimilated into a 3D dataset,
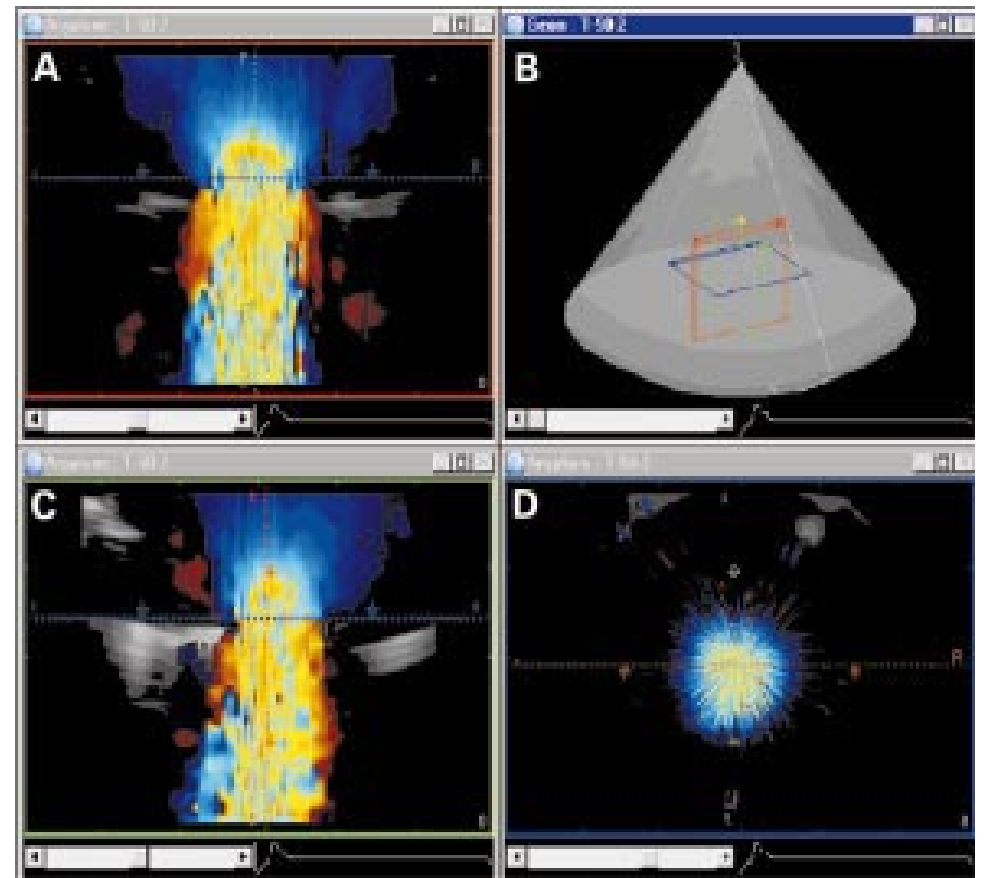

Figure 3 Localisation of the vena contracta in the $3 D$ dataset (in vitro acquisition). Colour Doppler flow data have been acquired parallel to flow. $(A)$ and $(C)$ show direction of flow from top to bottom in the two. (D) A perpendicular (blue cutplane) cut through the narrowest portion of the regurgitant jet, the vena contracta. most commonly through processing by a dedicated $3 \mathrm{DE}$ workstation.

Colour Doppler data may be incorporated into the dataset in several ways:

- as a component of the analogue composite video signal, with colours digitised to $\mathrm{red} / \mathrm{green} / \mathrm{blue}$ assignments for representation in the reconstructed dataset

- as digital scan converted and colour encoded raster data

- as digital velocity assignments derived from raw scan-line data, before the application of any scan conversion algorithms.

The acquisition of digital velocity data for 3D datasets facilitates data transfer and storage, since raw rather than scan converted data are involved. Doppler calibration is also more accurate than for 3D images made up of scan converted or colour video data. The major advantage however is that full velocity information is retained in the dataset, providing efficient, reliable, and flexible velocity reconstruction in $3 \mathrm{D}$ space. Firstly, this means that the data may be displayed in a variety of formats, allowing the operator to view the reconstructed flow using familiar colour maps similar to those in use on conventional 2D systems. Secondly, as will be discussed shortly, velocity features can be delineated and flow volumes and rates directly computed from these digital velocities. In those datasets generated from scan converted or analogue video data, velocity values must be reassigned to the colour values using the appropriate look-up tables.

3D representation of cardiac flow events Surface rendering techniques may be applied to colour Doppler datasets to generate images of regurgitant jets and flow convergence regions in three dimensional space (fig 2). In addition, manipulation of cutplanes through the dataset allows the vena contracta (the narrowest cross section of the regurgitant jet, 

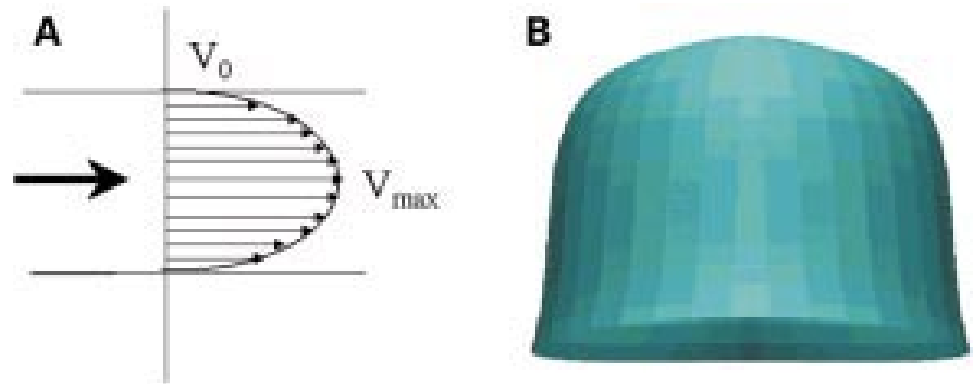

Figure 4 (A) Example of laminar flow pattern, such as might be encountered in the ventricular outflow tracts or the great vessels. (B) $3 D$ reconstruction of a laminar flow profile (in vitro acquisition).

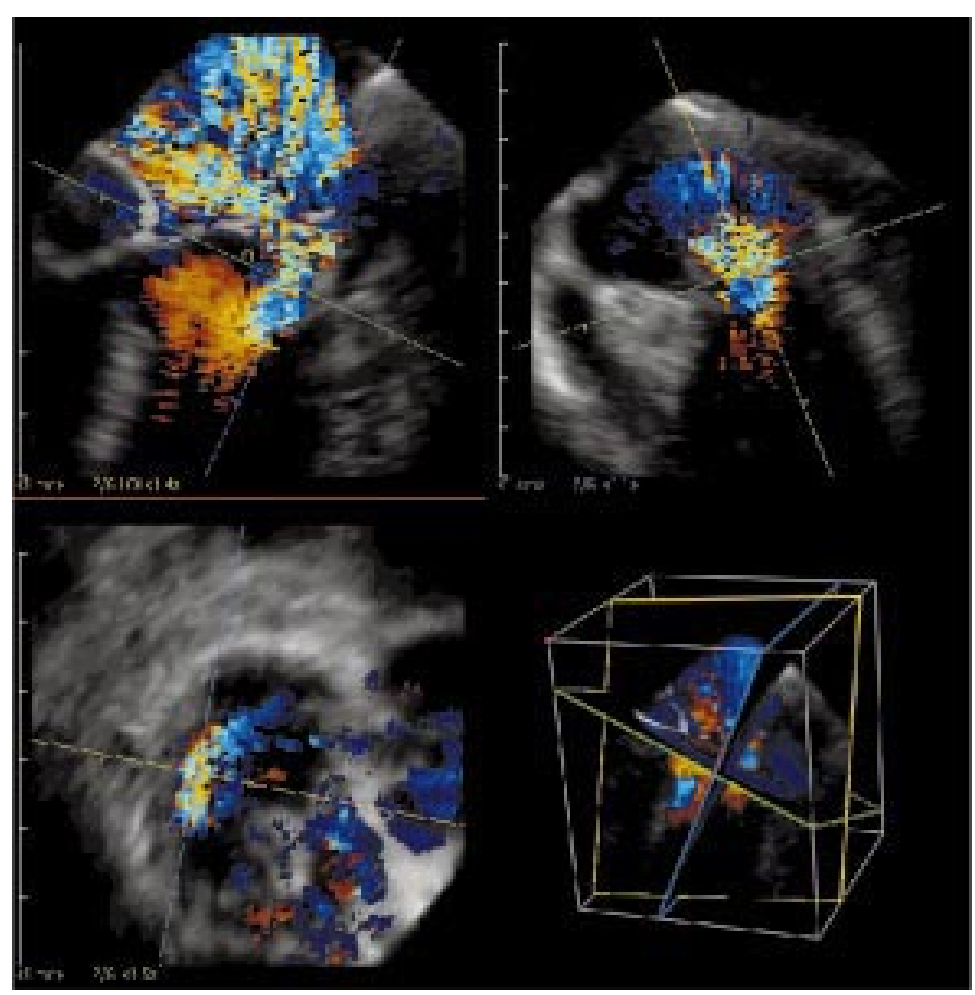

Figure 5 Identification of the vena contracta in a case of anterior mitral valve leaflet prolapse. In spite of the eccentricity of the jet, the cutplanes can still be manipulated to produce a cross section of the vena contracta (bottom left panel). It is then a simple process to measure its area. This should give an approximation of the regurgitant orifice area.

usually encountered at or in the immediate vicinity of the regurgitant orifice) to be precisely located (fig 3). Measurement of the jet cross section at this point should provide a reasonable approximation of the effective regurgitant orifice area. Such a technique highlights a further advantage of 3DE. Colour Doppler data must be acquired in an orientation parallel to flow to preserve the accuracy of measured velocity values. For conventional $2 \mathrm{D}$ colour Doppler, imaging perpendicular to flow to make cross sectional vena contracta measurements may lead to erroneous velocity signals due to the Doppler angle effect. 3DE allows velocity data to be acquired in a direction parallel to flow, and subsequent flow cross section measurements to be made perpendicular to flow within the $3 \mathrm{D}$ dataset without loss of accuracy of velocity information.

Of major importance, these features of $3 \mathrm{DE}$ (parallel acquisition with accurate digital velocity solutions) prove most advantageous in the assessment of laminar flows, such as might be encountered in the ventricular outflow tracts or great vessels. Flow in these structures travels in a series of laminar, concentric shells, with flow velocities being greatest in the centre of the vessel and approaching zero at the edges. ${ }^{25} 26$ This makes quantification of flow difficult, especially in the case of skewed flow patterns. 2DE methods for the quantification of outflow tract or great vessel flows involve pulsed wave Doppler sampling in the centre of the vessel and an assumption that the velocity measured is representative of the entire cross sectional flow profile..$^{12}{ }^{13}$ In the case of laminar flows, particularly if these are skewed, this may well not be the case. ${ }^{28}$ More sophisticated digital 2D methods exist which can characterise flow velocity profiles in two dimensional space-for example, Automated Cardiac Output Measurement, Toshiba Imaging Systems ${ }^{29-32}$, but assumptions must still be made regarding the morphology of the entire cross sectional flow profile in question. To reliably analyse laminar flow patterns, interrogation of the entire flow cross section is required. This is obviously not possible using conventional $2 \mathrm{D}$ imaging (because it would be necessary to image perpendicular to the angle of flow). Through its ability to acquire flow velocity data parallel to flow and subsequently analyse the resulting dataset in any plane, $3 \mathrm{DE}$ should permit full characterisation of the entire cross sectional velocity profile (fig 4 ). This should also allow more accurate quantification of absolute flow volume.

\section{Specific review of 3DE flow} quantification techniques

The 2D methods for flow quantification described in table 1 can also be applied to $3 \mathrm{D}$ datasets. Their accuracy is currently under investigation both in the in vitro and in vivo settings.

\section{D JET VOLUME CALCULATION}

Calculation of jet volume may overcome some of the problems encountered with $2 \mathrm{D}$ jet area methods. In the case of eccentric, wall adherent jets, calculation of jet volume might be a more reliable quantitative technique than jet area, since the latter is based on sections through the thin portion of the jet. This remains to be proved, however. Jet volume calculations will of course be influenced by the same machine factors as $2 \mathrm{D}$ jet area measurements, and preliminary results suggest that this may limit their usefulness. ${ }^{33}$

VENA CONTRACTA CROSS SECTIONAL AREA

The ability of 3DE to acquire flow velocity data parallel to flow and make subsequent flow computations in any plane has already been discussed. In addition, 3DE allows the user to navigate through a dataset and accurately pinpoint the position of the vena contracta, even if the jet itself is eccentrically positioned (fig 5). Area measurements can then easily be made. Even early 3D vena contracta studies, where flow events were imaged in grey scale, demonstrated the accuracy of this method. ${ }^{34}$ 


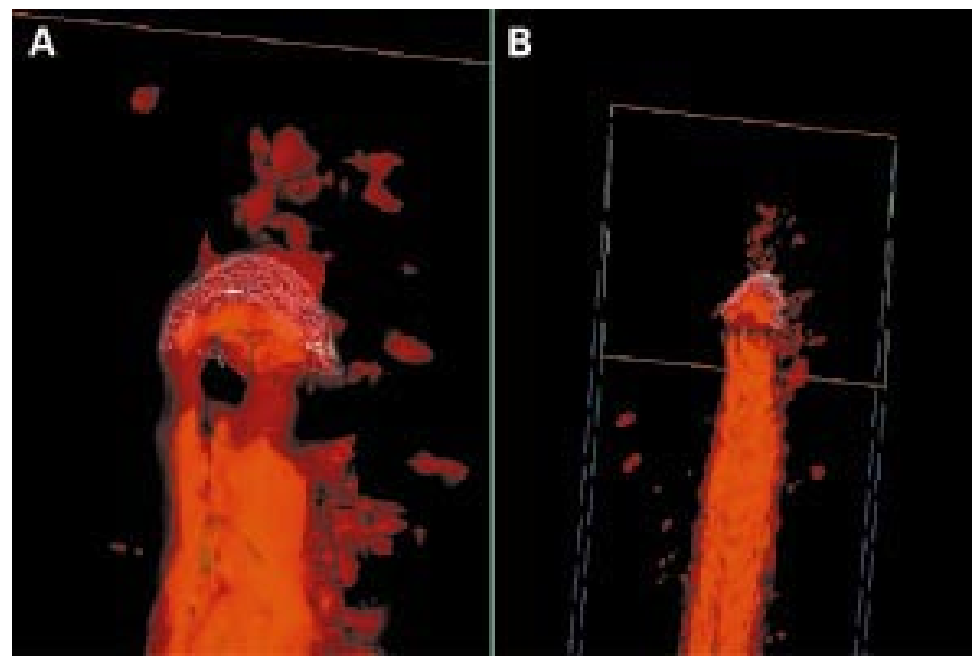

Figure 6 Calculation of the flow convergence region surface area in the $3 D$ dataset. $(A)$ is a magnified view of $(B)$. A wire model framework has been fitted to the surface of the flow convergence region. Surface area computations are made using this model. Note that no geometric assumptions are involved in the measurement of the surface area.

\section{Flow convergence region surface area computation}

The flow convergence region (FCR) is an isovelocity boundary within the flow field approaching a regurgitant orifice. Acceleration of flow towards the orifice occurs in an organised fashion, and as flow velocities exceed the aliasing limit an abrupt colour change occurs. Measurement of the area of this isovelocity surface is an integral part of FCR based flow rate and regurgitant orifice area computations. Since $3 \mathrm{DE}$ displays the entire FCR, a more accurate assessment of its area can be made without the need to make assumptions regarding its shape (fig 6). 3DE flow convergence based methods have been shown to accurately predict flow rate and regurgitant orifice area. ${ }^{35}$ Moreover, the retention of velocity assignments in digital Doppler 3DE allows user defined isovelocity regions to be extracted automatically from the dataset.

\section{Quantification of cardiac output and great vessel flows}

As indicated above, accurate quantification of flow through larger vessels, such as the ventricular outflow tracts or the great vessels, requires a method which can fully characterise complex and dynamically changing cross sectional flows, irrespective of their geometry. We have developed a digital 3D colour Doppler method which computes flow rates and stroke volumes through the spatiotemporal integration of the individual velocity vectors making up the flow cross section within the dataset. The technique is displayed in fig 7 and is described in detail elsewhere. ${ }^{37}$ In vitro studies using expansile rubber tubes have shown this to be an accurate method for calculation of instantaneous flow rates and stroke volumes. In addition to permitting full characterisation of the flow profile in three dimensional space, another advantage of this method is that changes in the area of cross sectional flow can be tracked as the vessel expands and contracts during the cardiac cycle. Applying such a method to flow through the ventricular outflow tracts, for example, should allow direct computation of cardiac output.

\section{Conclusion}

Continuing improvements in computing power and sophistication have led to the development of 3D workstations which can acquire and produce $3 \mathrm{D}$ datasets in a matter of minutes. Real time 3D colour Doppler imaging for clinical use is on the horizon. The accuracy of $3 \mathrm{DE}$
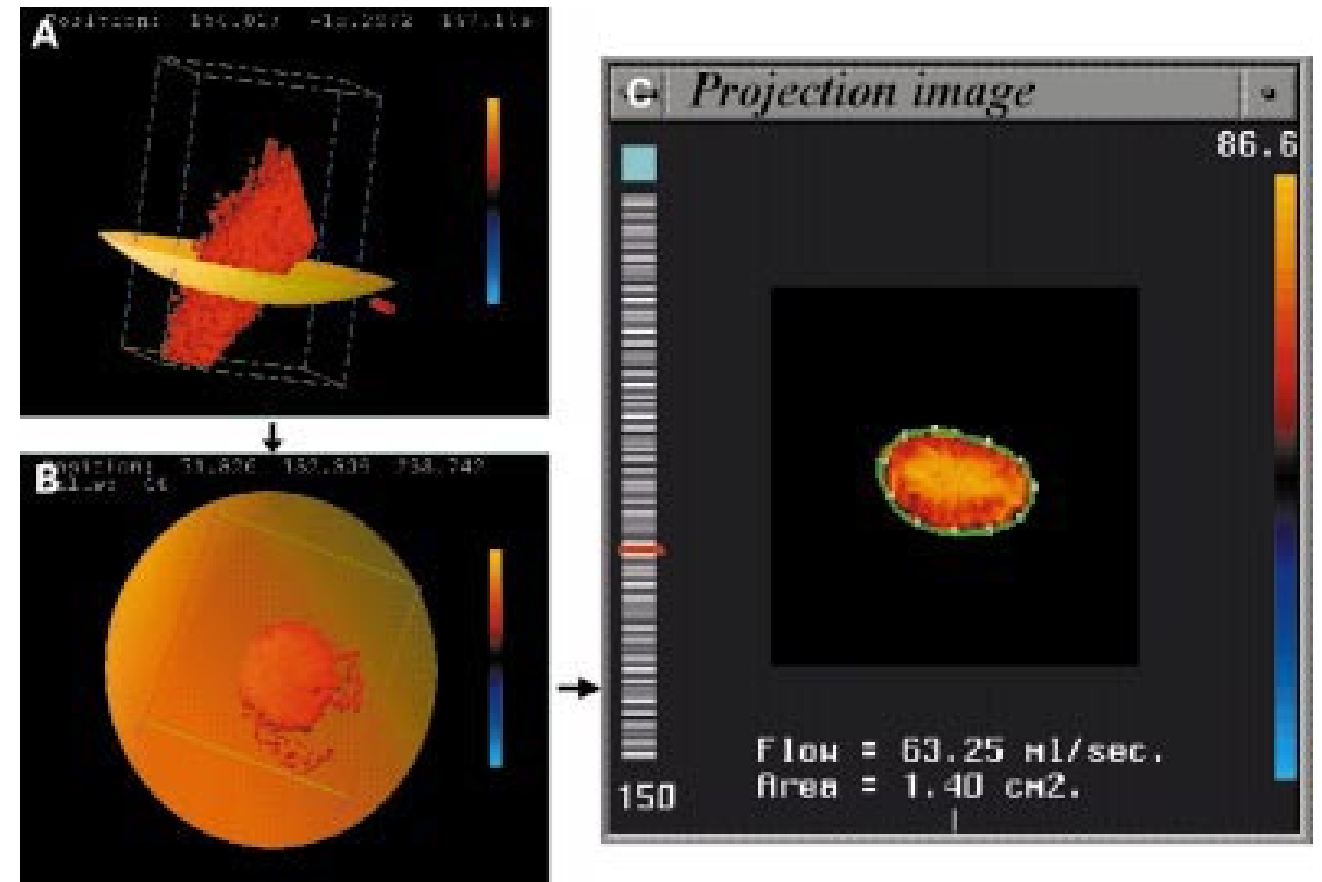

Figure 7 Computation of instantaneous flow rate from a digital 3D Doppler dataset, acquired from an in vitro tube model of laminar flow. The colour flow cross section is identified within the dataset (A), (B). The computer performs a spatial integration of the individual velocity vectors contained within the flow cross-section $(C)$ to generate an instantaneous flow rate. 
(and its superiority over conventional 2D methods) for the measurement of cardiac chamber volume has already been clearly shown. While $3 \mathrm{DE}$ flow quantification is at present in its infancy, the development and evaluation of new methods such as those described above are proceeding rapidly. We can be optimistic that in the not too distant future $3 \mathrm{DE}$ will provide a truly non-invasive method for the accurate quantification of cardiac output and for the reliable and reproducible assessment of valvar regurgitation.

1 Miyatake K, Izumi S, Okamoto M, et al. Semiquantitative grading of severity of mitral regurgitation by realtime twodimensional Doppler flow imaging technique. $\mathcal{f} \mathrm{Am} \mathrm{Coll}$ Cardiol 1986;7:82-8.

2 Helmcke F, Nanda NC, Hsuing MC, et al. Color Doppler assessment of mitral regurgitation with orthogonal planes. assessment of mitral regurgitation 1987;75:175-83.

3 Spain MG, Smith MD, Grayburn PA, et al. Quantitative assessment of mitral regurgitation by Doppler color flow imaging: angiographic and hemodynamic correlations. $\mathcal{f}$ Am Coll Cardiol 1989;13:585-90.

4 Bargiggia GS, Tronconi L, Sahn DJ, et al. A new method for quantitation of mitral regurgitation based on color flow Doppler imaging of flow convergence proximal to regurgitant orifice. Circulation 1991;84:1481-9.

5 Giesler M, Grossmann G, Schmidt A, et al. Color Doppler echocardiographic determination of mitral regurgitant flow from the proximal velocity profile of the flow convergence region. Am f Cardiol 1993;71:217-24.

6 Shiota T, Jones M, Yamada I, et al. Effective regurgitant orifice area by the color Doppler flow convergence method for
evaluating the severity of chronic aortic regurgitation. An animal study. Circulation 1996;93:594-602.

7 Fehske W, Omran H, Manz M, et al. Color-coded Doppler imaging of the vena contracta as a basis for quantification imaging of the vena contracta as a basis for quantification

8 Grayburn PA, Fehske W, Omran H, et al. Multiplane transesophageal echocardiographic assessment of mitral regurgitation by Doppler color flow mapping of the vena contracta. Am f Cardiol 1994;74:912-17.

9 Ishii M, Jones M, Shiota T, et al. Quantifying aortic regurgitation by using the color Doppler-imaged vena contracta: chronic animal model study. Circulation 1997;96:2009-15.

10 Borras X, Carreras F, Auge JM, et al. Prospective validation of detection and quantitative assessment of chronic aortic regurgitation by a combined echocardiographic and Doppler method. I Am Soc Echocardiogr 1988;1:422-9.

11 Dujardin KS, Enriquez-Sarano M, Bailey KR, et al. Grading of mitral regurgitation by quantitative Doppler echocardiography: calibration by left ventricular angiography in routine clinical practice. Circulation 1997;96: graphy 15 .

12 Ascah KJ, Stewart WJ, Levine RA, et al. Dopplerechocardiographic assessment of cardiac output. Radio echocardiographic assessment

13 Lee W, Rokey R, Cotton DB. Noninvasive maternal stroke volume and cardiac output determinations by pulsed Doppler echocardiography. Am F Obstet Gynecol 1988;158:50510.

14 De Castro S, Yao J, Pandian NG. Three-dimensional echocardiography: clinical relevance and application. $A m f$ Cardiol 1998;81:96G-102G.

5 Roelandt JR, Yao J, Kasprzak JD. Three-dimensional echocardiography. Curr Opin Cardiol 1998;13:386-96.

16 Roelandt JR. Three-dimensional echocardiography: new views from old windows. Br Heart $\mathcal{f}$ 1995;74:4-6.

17 Nosir YF, Stoker J, Kasprzak JD, et al. Paraplane analysis from precordial three-dimensional echocardiographic data sets for rapid and accurate quantification of left ventricular volume and function: a comparison with magnetic resonance imaging. Am Heart f 1999;137:134-43.
18 Jiang L, Siu SC, Handschumacher MD, et al. Threedimensional echocardiography: in vivo validation for right ventricular volume and function. Circulation 1994;89: 2342-50.

19 Papavassiliou DP, Parks WJ, Hopkins KL, et al. Threedimensional echocardiographic measurement of right ventricular volume in children with congenital heart disease validated by magnetic resonance imaging. I $\mathrm{Am}$ Soc Echocardiogr 1998;11:770-7.

20 Pini R, Giannazzo G, Di Bari M, et al. Transthoracic threedimensional echocardiographic reconstruction of left and right ventricles: in vitro validation and 3D;comparison with right ventricles: in vitro validation and 3D;comparison with
magnetic resonance imaging. Am Heart f 1997;133:221-9.

21 Levine RA, Weyman AE, Handschumacher MD. Threedimensional echocardiography: techniques and applications. Am f Cardiol 1992;69:121H-30H

22 Irvine $\mathrm{T}$, Derrick $\mathrm{G}$, Morris $\mathrm{D}$, et al. Three-dimensional echocardiographic reconstruction of mitral valve color Doppler flow events. Am 7 Cardiol 1900;84:1103-6.

23 Smith SW, Henry G, Pavy HG, et al. High-speed ultrasound volumetric imaging system-Part I: transducer design and beam steering. IEEE Trans Ultrason Ferroelectr Freq Contr 1991;38:100-8.

24 von Ramm OT, Smith SW, Pavy HG. High-speed ultrasound volumetric imaging system-Part II: parallel processing and image display. IEEE Trans Ultrason Ferroelectr Freq Contr 1991;38:109-15.

25 Yoganathan AP, Cape EG, Sung HW, et al. Review of hydrodynamic principles for the cardiologist: applications to the study of blood flow and jets by imaging techniques. $7 \mathrm{Am}$ Coll Cardiol 1988;12:1344-53.

26 Stein PD, Sabbah HN. Nature of flow in large arteries. Monogr Atheroscler 1990;15:54-62.

27 Robson SC, Murray A, Peart I, et al. Reproducibility of cardiac output measurement by cross sectional and Doppler echocardiography. Br Heart f 1988;59:680-4.

28 Zhou YQ, Faerestrand S, Matre $\mathrm{K}$, et al. Velocity distributions in the left ventricular outflow tract and the aortic anulus measured with Doppler colour flow mapping in normal subjects. Eur Heart f 1993;14:1179-88.

29 Sun JP, Pu M, Fouad FM, et al. Automated cardiac output measurement by spatiotemporal integration of color Doppler data. In vitro and clinical validation. Circulation 1997;95:932-9.

30 Aida S, Shiota T, Tsujino $\mathrm{H}$, et al. Quantification of aortic regurgitant volume by a newly developed automated cardiac flow measurement method: an in vitro study. $\mathcal{F} \mathrm{Am}$ Soc Echocardiogr 1998;11:874-81.

31 Hozumi T, Yoshida K, Akasaka $\mathrm{T}$, et al. Automated assessment of mitral regurgitant volume and regurgitant fraction by a newly developed digital color Doppler velocity profile integration method. Am f Cardiol 1997;80:132530.

32 Van Camp G, Carlier S, Cosyns B, et al. Quantification of mitral regurgitation by the automated cardiac output method: an in vitro and in vivo study. $\mathcal{f} \mathrm{Am}$ Soc Echocardiogr 1998;11:643-51.

33 Yao J, Masani ND, Cao QL, et al. Clinical application of transthoracic volume-rendered three-dimensional echocardiography in the assessment of mitral regurgitation. $\mathrm{Am}$ f Cardiol 1998;82:189-96.

34 Mori Y, Shiota T, Jones M, et al. Three-dimensional reconstruction of the color Doppler-imaged vena contracta for quantifying aortic regurgitation. Studies in a chronic animal model. Circulation 1999;99:1611-17.

35 Shiota $\mathrm{T}$, Sinclair B, Ishii $\mathrm{M}$, et al. Three-dimensional reconstruction of color Doppler flow convergence regions and regurgitant jets: an in vitro quantitative study. $\mathcal{F} \mathrm{Am}$ Coll Cardiol 1996;27:1511-18.

36 Shiota T, Jones M, Delabays A, et al. Direct measurement of three-dimensionally reconstructed flow convergence surface area and regurgitant flow in aortic regurgitation: in vitro and chronic animal model studies. Circulation 1997;96:3687-95

37 Irvine T, Li XN, Mori Y, et al. A digital 3D method for computing great artery flows: in vitro validation studies. $\mathcal{f} \mathrm{Am}$ Soc Echocardiogr [In press]. 